\title{
Online Via-Points Trajectory Generation for Reactive Manipulations*
}

\author{
Ran Zhao ${ }^{1,2}$, Daniel Sidobre ${ }^{1,2}$ and Wuwei $\mathrm{He}^{1,3}$
}

\begin{abstract}
In various circumstances, such as human-robot interactions and industrial processes, planning at trajectory level is very useful to produce better movement. In this paper we present a near time optimal approach to plan a trajectory joining via points in real time for robot manipulators. To limit the speed variation, the path is smoothed around via-points in a limited area. The concept of online trajectory generation enables systems to react instantaneously at control level to unforeseen events. Simulation and real-world experimental results carried out on a KUKA Light-Weight Robot arm are presented.
\end{abstract}

\section{INTRODUCTION}

Defining robot motion by trajectories has many advantages to describe feasible motions or human acceptable motions. While a path defined by a polygonal lines doesn't precise if the mobile must stop at via-points or smooth the corner, a trajectory describes precisely the motion. It is also well known that humans minimize the jerk of their movements [1] or that the bounding of the jerk and acceleration are necessary to limit the vibrations and the wear of mechanical parts during machining [2], [3]. In both case trajectories can describe the corresponding motion. Also, it is reasonable to replace paths by trajectories as the interface between planners and controllers, and to add a trajectory planner as an intermediate level in the software architecture.

To react to environment changes, the trajectory planning must be done in real time. Meanwhile, the robot needs to guarantee the human safety and the absence of collision. So the model for trajectory must allow fast computation and easy communication between the different components, including path planner, trajectory generator, collision checker and controller. To avoid the replan of an entire trajectory, the model must allow deforming locally a path or a trajectory. The controller must also accept to switch from an initial trajectory to follow to a new one.

In this paper we use third degree polynomial functions to describe trajectories and we propose an efficient method to generate trajectories. In the first stage, we suppose that a motion planner produces a polygonal line. From this polygonal line, we build a smoothed and feasible trajectory bounded in jerk, acceleration and velocity. This corresponds to the

\footnotetext{
*This work has been supported by the European Community's Seventh Framework Program FP7/2007-2013 "SAPHARI" under grant agreement no. 287513 and by the French National Research Agency project ANR-10CORD-0025 "ICARO".

${ }^{1,2}$ Ran Zhao and Daniel Sidobre are with CNRS, LAAS, 7 avenue du colonel Roche, F-31400 Toulouse, France; and Univ de Toulouse, UPS, LAAS, F-31400 Toulouse, France. ${ }^{1,3}$ Wuwei He is with CNRS, LAAS, 7 avenue du colonel Roche, F-31400 Toulouse, France; and Univ de Toulouse, LAAS, F-31400 Toulouse, France. ran.zhaodlaas.fr and daniel.sidobredlaas.fr and wuwei.hellaas.fr
}

type $\mathrm{V}$ trajectories in the classification proposed by Kroger [4]. As this operation modifies the initial free path, the new smoothed path must be checked for collision. Locally near a via-point, the path defines a subspace of dimension two and the trajectory proposed remains inside this subspace where the maximum error distance can be verified.

This paper focuses on the via-points trajectory planning for a service manipulator robot. We present related work in Section III. Soft Motion trajectory and the concept of online trajectory generation are detailed in Section III. Section IV describes a simple approach to build a smooth via-points trajectory. We explain how to manage the error in Section $V$. Simulation and experimental results are presented in Section VI using a 7-DOF arm.

\section{RELATED WORK}

Recent years have seen an increase in research in trajectory planning, especially related to the domains of Human-Robot Interactions (HRI) and high speed machining. Trajectory planning is commonly decomposed in two phases [5], firstly the selection of a path and then the generation of the trajectory, i.e. the building of a time evolution function along the path. A partial review for robotic is presented in Gaspareto et al. [6].

Probabilistic path planning methods [7] have had great success for robot motion, but they are not directly adapted to the changing robot environment of human-robot interaction. A lot of extensions like in [8]-[10] were proposed to improve reactivity. It should be noted that all these methods provide a polygonal path as output, which is impossible to follow at constant speed.

Broquère et al. [11] gives a solution to compute a time optimum trajectory bounded in jerk, acceleration and velocity for an axis joining any pairs of states defined by a position, a velocity and an acceleration. For a point to point movement in an $\mathrm{N}$-dimensional space, the time optimum straight-line motion is obtained by projecting the constraints on the line [12].

The trajectory planning problem can be solved in joint space or in the operational space, but kinematic models make the combined problems difficult. A method taking kinematic and process constraints into account for five-axis machining is presented in [13]. For more complex kinematics like 7-axis arm, the problem is still largely open to research.

In this paper we propose a solution to generate a trajectory from a polygonal path. The objective of the method is to build a time optimum trajectory while controlling the distance between the path and the trajectory. 


\section{Online Trajectory Generation (OTG)}

\section{A. Soft Motion Trajectory}

Trajectories are time functions defined in geometrical spaces, essentially Cartesian space and joint space. The rotations can be described using different coordinates system: quaternion, vector and angle etc. The books from Biagiotti [5] on one hand and the one from Kroger [4] on the other hand summarize background trajectory material.

We choose a particular series of $3^{\text {rd }}$ degree polynomial trajectories and name them as Soft Motion trajectories. A trajectory $\mathcal{T}$ is then a function of time defined as:

$$
\begin{aligned}
\mathcal{T}:\left[t_{I}, t_{F}\right] & \longrightarrow \mathbb{R}^{n} \\
t & \longmapsto \mathcal{T}(t)
\end{aligned}
$$

Where $\mathcal{T}(t)=Q(t)=\left({ }_{1} Q(t),{ }_{2} Q(t), \cdots,{ }_{n} Q(t)\right)^{T}$ for joint motions or $\mathcal{T}(t)=X(t)=\left({ }_{1} X(t),{ }_{2} X(t), \ldots,{ }_{n} X(t)\right)^{T}$ in Cartesian space from the time interval $\left[t_{I}, t_{F}\right]$ to $\mathbb{R}^{n}$. $n$ is the dimension of the motion space. Without loss of generality, we suppose that all ${ }_{j} Q(t)$ or ${ }_{j} X(t), 0 \leq j<n$ share the same time intervals and that $t_{I}=0$. The continuity class of the trajectory is $\mathcal{C}^{2}$.

A first OTG algorithm is able to calculate a trajectory at any time instant, which makes the system transfer from the current state to a target state. A state of motion at an instant $t_{i}$ is denoted as

$$
M_{i}=\left(P_{i}, V_{i}, A_{i}\right)
$$

where $P_{i}\left(P_{i}=Q\right.$ or $\left.P i=X\right)$ represents the position, $A_{i}$ the acceleration and $V_{i}$ the velocity at in instant $t_{i}$. Fig 1 illustrates the input and output values of OTG algorithm. We propose several trajectory generators which are capable of generating Soft Motion trajectories in one time cycle. The trajectory calculated is a type $\mathrm{V}$ trajectory in the classification proposed by Kröger, which satisfies:

$$
\begin{array}{rlrl}
V_{i} & \in \mathbb{R}^{n} & & \left|{ }_{j} V_{i}\right| \leq V_{\max } \\
A_{i} & \in \mathbb{R}^{n} & & { }_{j} A_{i} \mid \leq A_{\max } \\
J_{i} & \in \mathbb{R}^{n} & & { }_{j} J_{i} \mid \leq J_{\max } \\
P_{i} & \in \mathbb{R}^{n} &
\end{array}
$$

$J_{\max }, A_{\max }, V_{\max }$ are the kinematic constraints and $J_{i}$ is the jerk at time $t_{i}$. Therefore, at a discrete instant, the OTG algorithm can transfer motion states while not exceeding the given motion constraints.

\section{B. Trajectory Generators}

1) Phase Synchronized Trajectory Generation: Different solutions where proposed to generate monoaxial trajectories, but for the multiaxial case, generating trajectories with the same duration is not enough and a continuous phase synchronization is necessary to define the shape of the path. For example, to generate a straight line the ratio between the velocities of each axes must be constant.

Phase synchronization is the synchronization in position, velocity, acceleration and jerk spaces. It means that, given any instant of time, all variables must complete the same

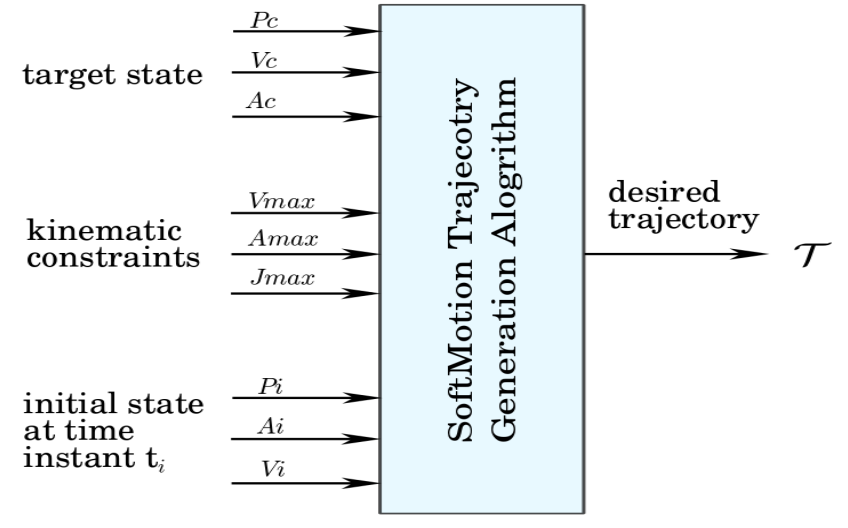

Fig. 1. Online trajectory generation algorithm

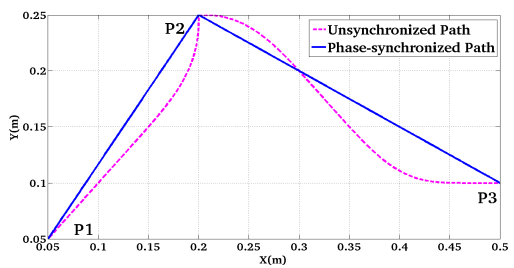

Fig. 2. Via-points motion: phase-synchronized and without synchronization

percentage of their trajectories. In a $n$ dimensional space, Frisoli et al. [14] defines the phase synchronization as:

$$
\frac{{ }_{i} X(t)-{ }_{i} X\left(t_{I}\right)}{{ }_{j} X(t)-{ }_{j} X\left(t_{I}\right)}=\lambda \quad \forall i, j \in[0, n-1], t \in\left[t_{I}, t_{F}\right]
$$

Where $\lambda$ is a constant. Fig 2 illustrates the path of a simple via-points motion in $2 \mathrm{D}$ space. $P_{1}, P_{2}, P_{3}$ are way points with zero velocities and accelerations. The difference between the phase-synchronized and unsynchronized trajectories are clearly depicted in Fig 3 . Phase-synchronized trajectories are very important for many real-world applications. For instance, phase-synchronized trajectories make sense when we want to modulate time w.r.t. cost values, which means, all axis stay at the same phase and slow/accelerate at the same time. In our case the shape of the curve is not defined, so we have to synchronize the initial and final state of motion and to define an acceptable curve.

2) Fixed Time Trajectory Generation: Planning a trajectory with initial position and final position now is well known, but planning in a fixed time is still an open problem in numerous cases. Thus to build a motion with predefined time, we propose two different methods by computing different parameters of the trajectory.

a) Three Segments Method: If we consider a portion of the trajectory $\mathcal{T}_{\text {in }}$ defined by an initial instant $t_{I}$ and a final instant $t_{F}$, the initial and final situations to connect are: $\left(X_{I}, V_{I}, A_{I}\right)$ and $\left(X_{F}, V_{F}, A_{F}\right)$. An interesting solution to connect this portion of trajectories is to define a sequence of three trajectory segments with constant jerk that bring the mobile from the initial situation to the final one in the 

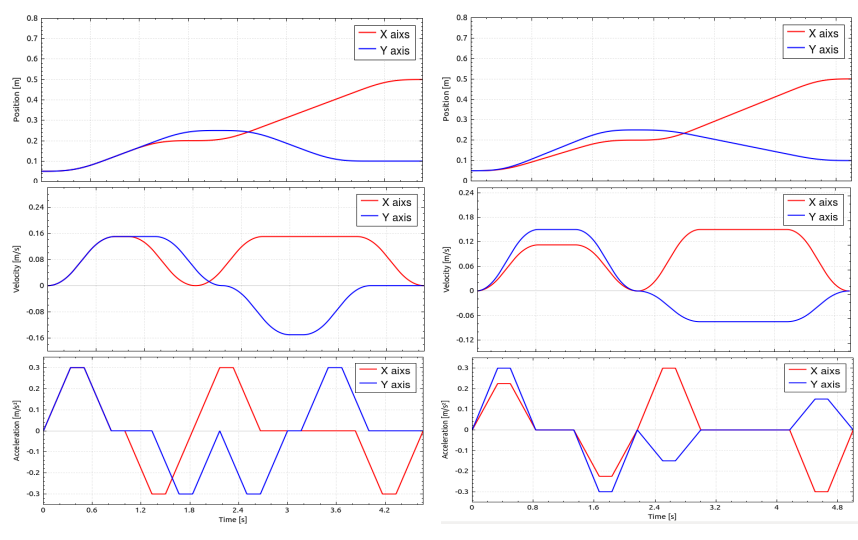

Fig. 3. Position, velocity and acceleration profile of $2 \mathrm{D}$ via points motion: without synchronization (left) and phase-synchronized (right).

time $T_{i m p}=t_{F}-t_{I}$. We choose three segments because we need a small number of segments and there is not always a solution with one or two segments.

The system to solve is then defined by 13 constraints: the initial and final situations (6 constraints), the continuity in position velocity and acceleration for the two switching situations and the time. Each segment of trajectory is defined by four parameters and one time. If we fix the three durations $T_{1}=T_{2}=T_{3}=\frac{T_{i m p}}{3}$, we obtain a system with 13 parameters where only the three jerks are unknown [15]. As the final control system is periodic with period $T$, the times $T_{i m p} / 3$ must be a multiple of the period $T$ and $T_{i m p}$ chosen to be a multiple of $3 T$.

b) Three Segments Method With Bounded Jerk: Three segments method solves the fixed time trajectory generation problem with fixed duration for each segment. However, it cannot guarantee the computed jerk is always bounded. Here we introduce a variant three segments method with defined jerk.

As for the three segments method, the system is also defined by 13 constraints. But here we fix the jerk on the first and third segment $\left|J_{1}\right|=\left|J_{3}\right|$, which have value bounded within the kinematic limits. Thus the unknown parameters in the system are $J_{2}$ and the three time durations. Thus we obtain the system of four equations with four parameters $\left(J_{2}\right.$, $T_{1}, T_{2}$ and $\left.T_{3}\right)$ :

$$
\begin{aligned}
A_{F} & =J_{3} T_{3}+A_{2} \\
V_{F} & =J_{3} \frac{T_{3}^{2}}{2}+A_{2} T_{3}+V_{2} \\
X_{F} & =J_{3} \frac{T_{3}^{3}}{6}+A_{2} \frac{T_{3}^{2}}{2}+V_{2} T_{3}+X_{2} \\
T_{i m p} & =T_{1}+T_{2}+T_{3}
\end{aligned}
$$

Where

$$
\begin{aligned}
A_{2}= & J_{2} T_{2}+J_{1} T_{1}+A_{I} \\
V_{2}= & J_{2} \frac{T_{2}^{2}}{2}+\left(J_{1} T_{1}+A_{I}\right) T_{2}+J_{1} \frac{T_{1}^{2}}{2}+A_{I} T_{1}+V_{I} \\
X_{2}= & J_{2} \frac{T_{2}^{3}}{6}+\left(J_{1} T_{1}+A_{I}\right) \frac{T_{2}^{2}}{2}+\left(J_{1} \frac{T_{1}^{2}}{2}+A_{I} T_{1}+V_{I}\right) T_{2} \\
& +J_{1} \frac{T_{1}^{3}}{6}+A_{I} \frac{T_{1}^{2}}{2}+V_{I} T_{1}+X_{I}
\end{aligned}
$$

To choose the value of jerks on each dimension, we take the velocity $V_{I}$ and $V_{F}$ into account. The jerks are fixed by $J_{1}=-J_{3}=J_{\max }$ when $V_{I}-V_{F}>0$, while $J_{1}=-J_{3}=$ $-J_{\max }$ when $V_{I}-V_{F}<0$. If $V_{I}-V_{F}=0$ then we compare the values of $A_{I}$ and $A_{F}$ instead.

Compared with the fixed time three segments method, the advantage of this method is that it can generate much softer motions since 2 of the 3 jerks are surely bounded. Meanwhile, if we follow a long path and make $J_{1}$ and $J_{3}$ saturated, we will get a near zero jerk and a big time $T_{2}$ on the second segment, that means we will have a long acceleration constant trajectory along the path, which is much more smoother than trajectory with changing acceleration.

\section{Building a Smoothing Motion Trajectory FROM WAY POINTS}

In this section, we begin with the two dimensional problem. Firstly, we present a solution to plan point-to-point (straight-line) trajectories that halt at each via-point where the direction of the path changes. Then we smooth the edges of the trajectory to produce smoother motion defined by kinematics conditions. Moreover, as the path is not predefined, different solutions exit for a vertex. We propose to compute a minimal time motion after defining the limits of the smoothed zone. Among the infinity of solution we choose one, which is simple to compute in real time.

\section{A. Time-optimal Straight-line Trajectory}

The first step is to convert a piecewise linear path to a time-optimal trajectory that stops at each via-point. In task space, straight-line paths are often required between the way points. The trajectory along a straight-line path should be a phase-synchronized motion [11].

We note the point-to-point trajectory as $\mathcal{T}_{p t p}$, it is computed by projecting the constraints of each axis on the straight-line and computing the minimum time necessary for this point to point movement. The time optimal solution of this monodimensional problem is then projected on the $n$ initial axis. For each segment of the trajectory, one of the velocity, acceleration, or jerk functions of the $n$ initial axes is saturated. The others are inside their validity domain. In other words, the motion is minimum time for one direction. In the other directions, the motions are conditioned by the minimum one. Repeating this strategy for each straight segment, we build the time optimal trajectory $\mathcal{T}_{p t p}$ that stops at each all via-points. 


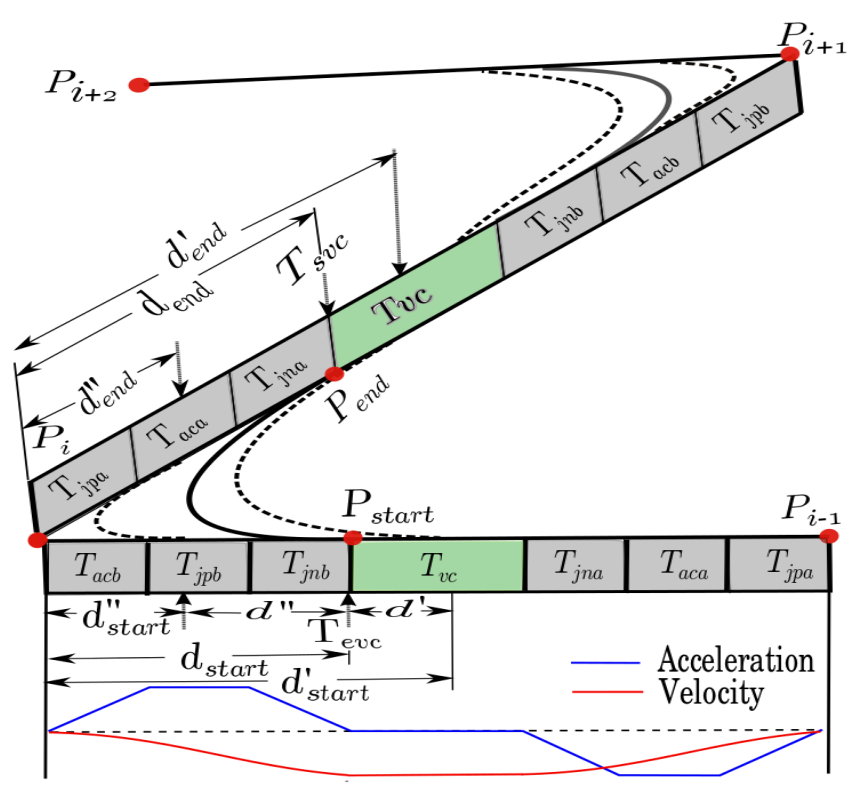

Fig. 4. Influence of the start and end points for the smooth area

\section{B. Smoothing a corner}

In the second step of the algorithm, we develop a solution to smooth the corners. We will discuss the case for 2D space and extend the solution to $3 \mathrm{D}$ in the next paragraph.

The point-to-point (straight-line) trajectory $\mathcal{T}_{p t p}$ obtained in the previous paragraph is feasible, but it is not satisfactory because the velocity varies greatly at each via-point to stop the motion. These stops can be avoided by allowing the trajectory to deviate slightly from the via-points by rounding the edges to smoothly travel near the point while changing the direction without stopping.

Without loss of generality we consider three adjacent points $\left(P_{i-1}, P_{i}, P_{i+1}\right)$ and the smoothing at the intermediate via-point $P_{i}$. Firstly, the two straight-line trajectories $\mathcal{T}_{P_{i-1} P_{i}}$ and $\mathcal{T}_{P_{i} P_{i+1}}$ are computed respectively. Then we choose two points $\left(P_{\text {start }}, P_{\text {end }}\right)$ based on the parameter $d$, which is the distance from the point $P_{i}$ on the trajectory. $\left(d_{\text {start }}, d_{\text {end }}\right)$ are used to denote the two distances and they defines the limits of the smoothed area. The possible choices of these two points are infinite, and the resulting trajectories can vary greatly due to the different choices, see Fig 4 . But since we wish a time optimal motion, a first simple idea is to keep the maximum velocity segment and smooth the area where the trajectory is not traveled at constant speed. Thence we choose two time instants: $T_{\text {evc }}$ is the end of velocity constant segment of $\mathcal{T}_{P_{i-1} P_{i}} ; T_{s v c}$ is the start of velocity constant segment of $\mathcal{T}_{P_{i} P_{i+1}}$, as shown in Fig 4 In a first case, $P_{\text {start }}$ and $P_{\text {end }}$ are defined by the kinematic conditions: $M_{\text {start }}=$ $\left(P_{e v c}, V_{e v c}, A_{e v c}\right)$ and $M_{e n d}=\left(P_{s v c}, V_{s v c}, A_{s v c}\right)$. As they are in the velocity constant segments, $A_{e v c}=A_{s v c}=0$ in this case. Then we compute the minimum time trajectory between $P_{\text {start }}$ and $P_{\text {end }}$ independently on the two axes using the 7 segment method, proposed by Broquère in [11]. The optimal time is the longer one of the two and the problem becomes to find a motion along the other axis that last the same time. Then the methods proposed in III-B.2 can be used to build trajectory $\mathcal{T}_{s}$ from $M_{\text {start }}$ to $M_{\text {end }}$. Thus, we have a trajectory $\mathcal{T}$ composed of 3 segments from $\mathcal{T}_{s}$ and 8 segments from $\mathcal{T}_{\text {ptp }}$.

However, an exception exits when the angle $\alpha$ formed by the 3 points is quite small $\left(\alpha<\alpha_{\text {lim }}, \alpha_{\text {lim }}\right.$ depends on the kinematic constraints), the previous solutions can not work any more because they will get either jerks much larger than the kinematic constraints or negative time. In this case, for $d \leq d_{\text {start }}$ the optimal time solution is to stop at the via points. So we propose to increase the distance by $d_{\text {start }}^{\prime}=$ $d_{\text {start }}+d^{\prime}$ to smooth the corner.

\section{Extension to $3 D$ problem}

Now we extend this solution to the three dimensional problem. Locally to a via point, the two adjacent straight segment defines a plane. Thus the 3-dimensional smoothing problem can be solved by projecting the constraints in this plane. For isotropic constraints in Cartesian space, constraints remain the same after the projection. Then we can easily apply the $2 \mathrm{D}$ trajectory generation approach to this $3 \mathrm{D}$ problem and finally project back the trajectory in the initial frame. However, for joint space problem, it is far more complex to project the constraints. One simple solution is to choose the minimum constraints among the projected constraints on each axis.

\section{MAnAging THE ERROR}

From a user point of view, managing the position error at via-points can be more important than defining the distance $d$ where smoothing begins. The trajectory error can be defined as the largest distance between the initial straight-line trajectory and the smoothed one. It represents how much the smooth trajectory deviate from the via-points. This approach is useful, for example, if the path planner provides some tube around the path where the motion is free of collision.

\section{A. Error Definition}

Considering the case of three adjacent points $P_{i-1}, P i, P_{i+1}$, see Fig.5, $\overrightarrow{\mathbf{n}_{i}}$ being the normal unit vectors to the straight line $P_{i-1} P_{i}$, and $\mathcal{T}_{s i}(t)$ the smoothed trajectory. Then the error $\mathcal{E}$ can be defined as:

$$
\begin{aligned}
& \mathcal{E}(t)=\min _{t \in\left[t_{I}, t_{F}\right]}\left(\left[\mathcal{T}_{s i}(t)-P_{i}\right] \cdot \overrightarrow{\mathbf{n}_{i}},\left[\mathcal{T}_{s i}(t)-P_{i}\right] \cdot \overrightarrow{\mathbf{n}_{i+1}}\right) \\
& \mathcal{E}=\max (\mathcal{E}(t))
\end{aligned}
$$

To compute this error, we introduce another parameter $\mathcal{E}_{v}$, which is represented by the minimum distance between the vertex $P_{i}$ and the trajectory $\mathcal{T}_{s i}(t)$ :

$$
\mathcal{E}_{v}=\min _{t \in\left[t_{I}, t_{F}\right]} d\left(P_{i}, \mathcal{T}_{s i}(t)\right)
$$

As the start and end points of the blend we choose locate at the symmetric segments on each straight-line trajectory, the 


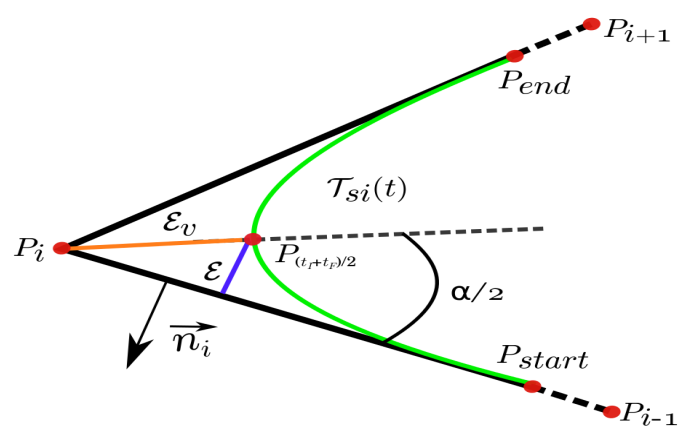

Fig. 5. Error between the smoothed trajectory and pre-planed path

error $\mathcal{E}_{v}$ happens at the bisector of the angle $\alpha$ formed by the 3 adjacent points, Therefore,

$$
\begin{aligned}
\mathcal{E}_{v} & =d\left(P_{i}, \frac{\mathcal{T}_{t_{I}+t_{F}}}{2}\right) \\
\mathcal{E} & =\mathcal{E}_{v} * \sin \frac{\alpha}{2}
\end{aligned}
$$

\section{B. Comply with Maximum Error}

We suppose that the task planner provides a maximum tolerable error $D$. A simple possibility is to change the distance $d$ to make the error $\mathcal{E} \leq D$, as shown in Fig 4 We introduce a parameter $\delta$, for a general case, it is defined as:

$$
\delta=\frac{d\left(P_{\text {start }}, P_{i}\right)}{d\left(P_{\text {Tevc }}, P_{i}\right)}
$$

Thus $\delta \in[0,1]$. But when $\alpha<20^{\circ}, P_{\text {start }}$ locates at the segment before $P_{\text {Tevc }}$, so in this case:

$$
\delta=\frac{d\left(P_{\text {start }}, P_{i}\right)}{d\left(P_{\text {Tevc }}, P_{i}\right)}-1
$$

Suppose $P_{\text {end }}^{i}$ is the end point of the previous blend and $P_{\text {start }}^{i+1}$ is the start point of the next one. To avoid the discontinuity, the distance $d$ of new points must satisfy:

$$
d_{\text {end }}^{i}+d_{\text {start }}^{i+1} \leq d\left(P_{i}, P_{i+1}\right)
$$

Then we can compute in a loop and get the $\delta_{\max }$ that makes $\mathcal{E} \leq D$ and also satisfies 16 .

\section{EXPERIMENTAL RESULTS}

\section{A. Simulation}

We set up a simulation environment with a collision checker ${ }^{1}$ In each time cycle, the collision checker checks the collision and each time a collision is detected, the system will replan the trajectory. The algorithm is shown in algorithm 1 In this simulation, we set $\delta=1$ to manage the error between the via-points and the smoothed trajectory because we aim at a time optimal motion.

Fig 6 shows the movement of the robot arm from an initial position $P_{i}$ to a final position $P_{f}$ with an obstacle. The robot successfully reaches the target position after two local trajectory deformations. It shall be noted that the robot is reactive, and the same trajectory can be used for a moving obstacle. Fig.7 presents the traveled trajectory along the
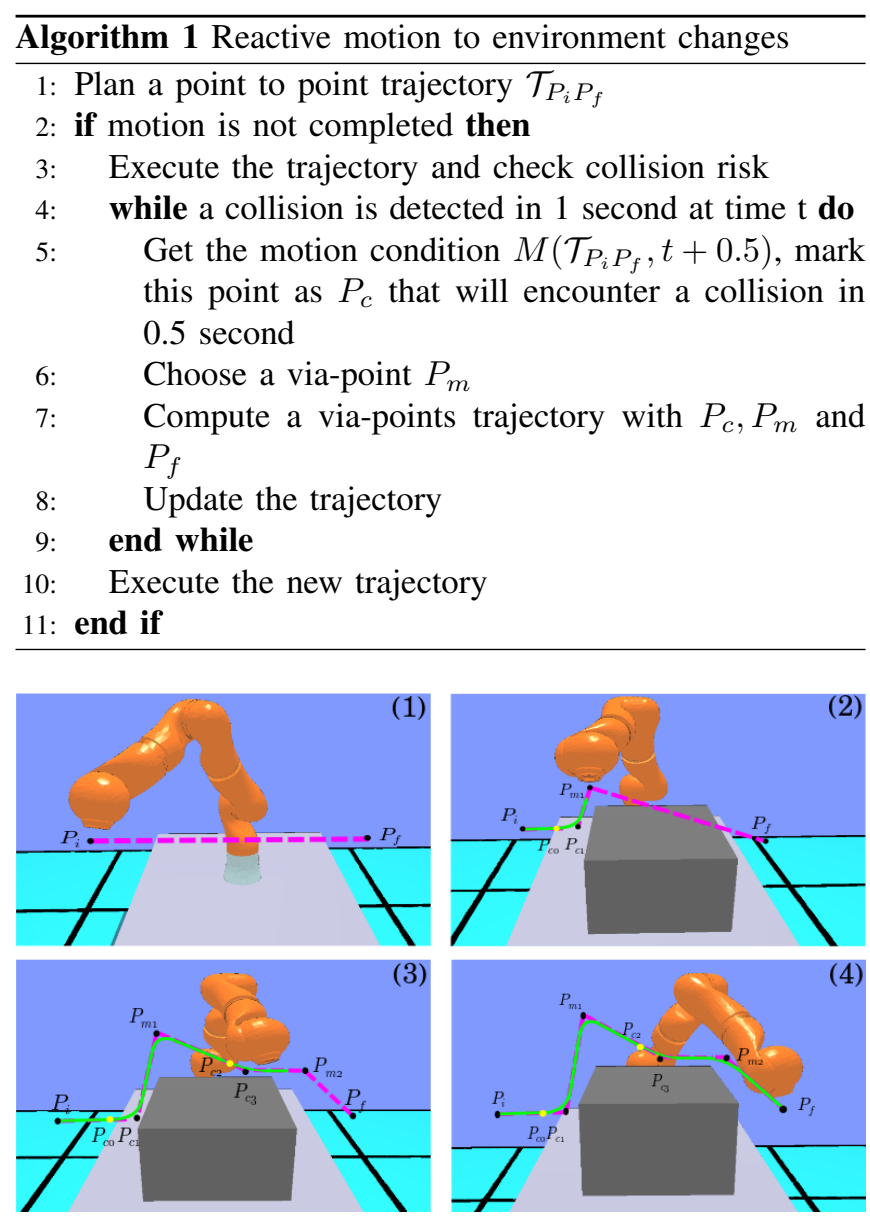

Fig. 6. A simulated move form an initial position to an final position with a static obstacle. The purple dashed line is the trajectory computed in advance. The green solid line is the real path which the robot follows. By adding two way points, the robot reaches the target position without collision and path replanning at the high level.

$\mathrm{Z}$ axis. The position curve shows how the trajectory deviate from the pre-planed one (straight line from the initial position to the final position) due to the predicted collision risk. In the first four segments, the executed trajectory coincides with the pre-planed one. At the collision prediction point 1 , the system switches to a new trajectory. The smooth velocity profile shows that the trajectory transition is instantaneous and continuous.

The computation for this Cartesian space trajectory with 6 via-points requires an average execution time of $1.2 \mathrm{~ms}$ on a Intel Core ${ }^{(T M)} 2$ Quad CPU $2.66 \mathrm{GHz}$ machine. It is fast enough for real time applications, such as visual servoing or reactions to other sensor events.

\section{B. Robot Experiments}

We also implemented the via-points trajectory generator on a KUKA light-weight robot IV [16], which was controlled through the Fast Research Interface [17]. The software control is developed using Open Robots tools: GenoM [18].

\footnotetext{
${ }^{1}$ http://robotpkg.openrobots.org/robotpkg/graphics/coldman-genom
} 


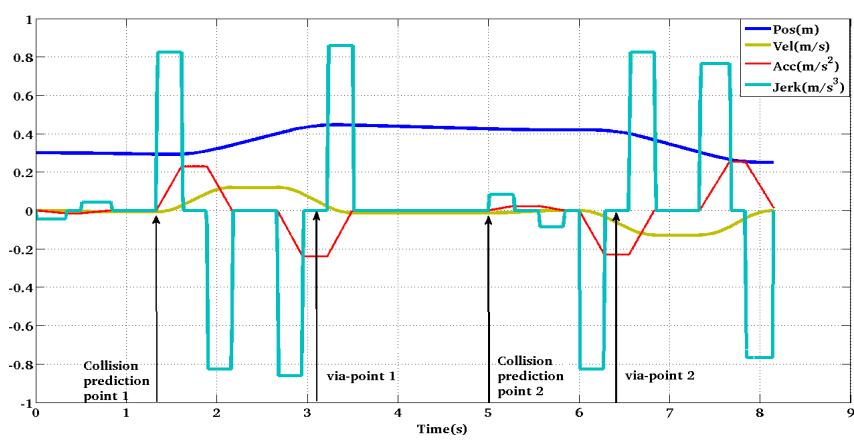

Fig. 7. The position, velocity, acceleration and jerk of online generated via-points trajectory on $Z$ axis

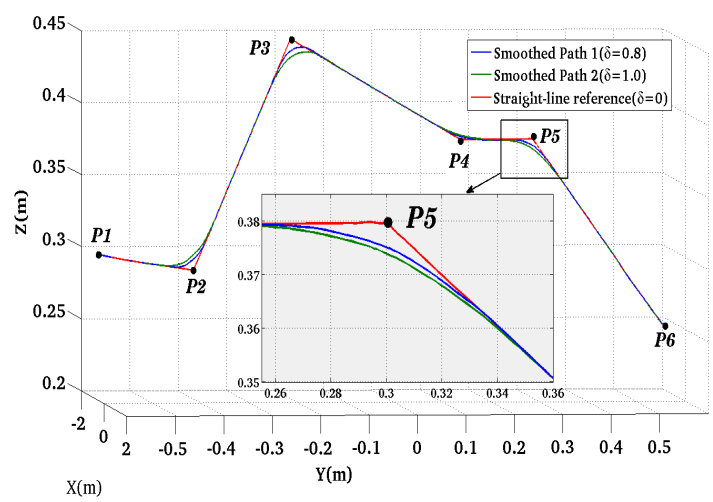

Fig. 8. Paths of the robot end effector with different $\mathcal{E}_{v}$

The sampling time is fixed to $10 \mathrm{~ms}$. The Pose of the manipulator's end effector is defined by seven independent Operational Coordinates. It is composed of a position vector $\mathbf{P}=[x, y, z]^{T}$ and by a quaternion $\mathbf{Q}=[n, \mathbf{q}]^{T}$, where $\mathbf{q}=[i, j, k]^{T}$. They give the position and the orientation of the final body in the reference frame. The linear and angular end-effector motion bounds are given in table $\mathrm{I}$.

TABLE I

ROBOT MOTION IS LIMITED IN JERK, ACCELERATION, AND VELOCITY

\begin{tabular}{|c|c|c|c|}
\hline & Jmax & Amax & Vmax \\
\hline Linear limits & $0.900 \mathrm{~m} / \mathrm{s}^{3}$ & $0.300 \mathrm{~m} / \mathrm{s}^{2}$ & $0.150 \mathrm{~m} / \mathrm{s}$ \\
\hline Angular limits & $0.600 \mathrm{rad} / \mathrm{s}^{3}$ & $0.200 \mathrm{rad} / \mathrm{s}^{2}$ & $0.100 \mathrm{rad} / \mathrm{s}$ \\
\hline
\end{tabular}

Fig 8 illustrates the the same path of the robot end effector as in the previous simulation. $\delta=1.0$ means the smoothed trajectory starts at the end of maximum velocity segment. While $\delta=0.8$ means the start points are closer to the intermediate points, which results in a smaller error. The straight-line path is a path with zero error, which can be represented by defining $\delta=0$.

\section{CONCLUSIONS AND FUTURE WORKS}

This paper proposed a solution to generate trajectories defined by via-points and a parameter defining the size of the smoothing area. Using projection of constraints, it can build trajectories in Cartesian space. Due to direct computation without optimization computation or randomized algorithms, the proposed solution takes short execution time and is able to be used online. Experimental results show the validity of the approach for real-time control, especially for online collision avoidance. We are going to integrate the viapoints generator for realizing a real reactive manipulation. It would also be interesting to introduce the kinematic model to take into account both joint and Cartesian constraints. New solutions for fixed time trajectory generation can be proposed.

\section{REFERENCES}

[1] T. Flash and N. Hogan. The coordination of arm movements: an experimentally confirmed mathematical model. Journal of neuroscience, 1985

[2] Jingyan Dong, PM Ferreira, and JA Stori. Feed-rate optimization with jerk constraints for generating minimum-time trajectories. International Journal of Machine Tools and Manufacture, 47(12):1941-1955, 2007.

[3] A Zabel, H Müller, M Stautner, and P Kersting. Improvement of machine tool movement for simultaneous five-axes milling. In CIRP international seminar on intelligent computation in manufacturing engineering, pages 159-64, 2006.

[4] T. Kröger. On-Line Trajectory Generation in Robotic Systems, volume 58 of Springer Tracts in Advanced Robotics. Springer, Berlin, Heidelberg, Germany, first edition, jan 2010.

[5] L. Biagiotti and C. Melchiorri. Trajectory Planning for Automatic Machines and Robots. Springer, November 2008.

[6] Alessandro Gasparetto, Paolo Boscariol, Albano Lanzutti, and Renato Vidoni. Trajectory planning in robotics. Mathematics in Computer Science, 6(3):269-279, 2012.

[7] S. M. LaValle and J. Kuffner. Rapidly-exploring random trees: Progress and prospects. In Workshop on the Algorithmic Foundations of Robotics, 2001.

[8] Léonard Jaillet and Thierry Siméon. Path deformation roadmaps: Compact graphs with useful cycles for motion planning. The International Journal of Robotics Research, 27(11-12):1175-1188, 2008.

[9] Léonard Jaillet, Juan Cortés, and Thierry Siméon. Sampling-based path planning on configuration-space costmaps. Robotics, IEEE Transactions on, 26(4):635-646, 2010.

[10] Santiago Garrido, Luis Moreno, and Dolores Blanco. Exploration of a cluttered environment using voronoi transform and fast marching. Robotics and Autonomous Systems, 56(12):1069-1081, 2008.

[11] Xavier Broquere, Daniel Sidobre, and Ignacio Herrera-Aguilar. Soft motion trajectory planner for service manipulator robot. Intelligent Robots and Systems, 2008. IROS 2008. IEEE/RSJ International Conference on, pages 2808-2813, Sept. 2008.

[12] Daniel Sidobre and Wuwei He. Online task space trajectory generation. In Workshop on Robot Motion Planning Online, Reactive, and in Real-time, 2012.

[13] Hongyao Shen, Jianzhong Fu, and Zhiwei Lin. Five-axis trajectory generation based on kinematic constraints and optimisation. International Journal of Computer Integrated Manufacturing, (ahead-ofprint):1-12, 2014.

[14] A.Frisoli, C.Loconsole, R.Bartalucci, and M.Bergamasco. A new bounded jerk on-line trajectory planning for mimicking human movements in robot-aided neurorehabilitation. Robotics and Autonomous Systems, 2013.

[15] X. Broquère and D. Sidobre. From motion planning to trajectory control with bounded jerk for service manipulator robots. In IEEE Int. Conf. Robot. And Autom., 2010.

[16] KUKA Roboter GmbH. http: / / www.kuka.com/en/company/ group

[17] G. Schreiber, A. Stemmer, and R. Bischoff. The fast research interface for the kuka lightweight robot. In Proc. of the IEEE ICRA 2010 Workshop on ICRA 2010 Workshop on Innovative Robot Control Architectures for Demanding (Research) Applications - How to Modify and Enhance Commercial Controllers, pages 15-21, 2010.

[18] R. Chatila S. Fleury, M. Herrb. Genom: A tool for the specification and the implementation of operating modules in a dist ributed robot architecture. In EEE/RSJ International Conference on Intelligent Robots and Systems, pages 842-848, 1997. 